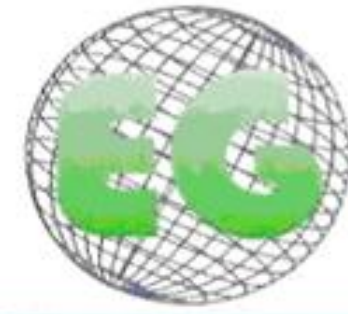

No38

\title{
Entornos educativos saludables de los estudiantes universitarios de enfermería
}

Healthy educational settings and their correlation with academic performance of nursing students

\section{*Gómez, Patricia Fabiana **Cometto, María Cristina ${ }^{* * *}$ Cerino, Sandra \\ "Cátedra de Salud Mental y Psiquiatría. E-mail: patriciafabianag@gmail.com **átedra de Alto Riesgo. ****átedra de Salud Mental y Psiquiatría. Escuela de Enfermería, Facultad de Ciencias Médicas, Universidad Nacional de Córdoba. Argentina.}

Palabras clave: entorno saludable; rendimiento académico; enfermería

.Keywords: healthy environment; academic performance; Nursing

\section{RESUMEN}

Introducción: La presente investigación tuvo como objetivo determinar los entornos de estudio y aprendizaje que poseen los estudiantes de enfermería de la Universidad Nacional de Córdoba.

Material y método: Estudio observacional-analítico, período 2011. Universo, estudiantes que cursan la licenciatura. Muestra estratificada, aleatoria. Técnica encuesta auto administrada. Fuente primaria.

Resultados: La población estuvo compuesta por 290 alumnos, el $46 \%$ poseen entre 18 y 25 años, el $44 \%$ entre 26 y $40 ; 83 \%$ mujeres; $68 \%$ solteros; el $76 \%$ convive con la familia, y el $69 \%$ posee familiares a cargo; el $56 \%$ trabaja y el $44 \%$ recibe ayuda de los padres o beca.

Al analizar el entorno en la dimensión organización y gestión, en la facilitación de materiales, se destaca su disponibilidad en más del 70\%. En la planta física el 50\% respondieron que no es adecuada. Los alumnos reciben apoyo en sus distintas variantes pedagógicas un $75 \%$. En la planificación de cátedras el $78 \%$ que no existe. El $52 \%$ respondió que la conducción en el período estudiado no demuestra habilidad para la gestión. Con respecto al aspecto vincular académico los estudiantes no se sienten escuchados en la institución en un $65 \%$, respondiendo que la comunicación entre pares es poco o medianamente colaborativa en un $59 \%$. El $78 \%$ de los estudiantes afirman que no existe comunicación entre los dos ciclos. Por último a la dimensión de la participación estudiantil en relación al centro de estudiantes el $92 \%$ respondieron que no participan activamente y el $59 \%$ afirman que no promueve un entorno saludable.

Conclusión: La organización y gestión, y la comunicación son identificadas negativamente. Consideramos que resta diseñar elementos que visibilicen esta problemática como central en la política de gestión académica, para construir estrategias conjuntas de resolución. 


\section{ABSTRACT}

Introduction: This research aimed to determine the study and learning environments that have nursing students of the National University of Córdoba.

Methods: Observational - analytical study, period 2011 Universe all students pursuing the degree. Sample stratified random. Technical self-administered survey. Primary source.

Results: The population consisted of 290 students studying different subjects of the degree. $46 \%$ of students have between 18 and 25 years, $44 \%$ between 26 and 40; 83\% female; $68 \%$ were single; $76 \%$ live with family, and $69 \%$ have dependents; $56 \%$ work and $44 \%$ receive help from parents or scholarship.

When analyzing the environment in the organization and management dimension, in facilitating learning materials in-house services discipline highlights its availability in more than $70 \%$. In the variable physical plant over $50 \%$ responded that it is not appropriate. Students are supported in their different pedagogical variations in values higher than $75 \%$ As formulation processes complaints, $75 \%$ responded that their claims are not heard: by managers in planning Chairs respondents $78 \%$ do not exists a negative impact on all the subdomains. $52 \%$ of respondents said that driving during the study period shows no ability to manage. Regarding the aspect linking academic students do not feel heard in the institution by $65 \%$, responding to peer communication is low and intermediate collaboratively by 59 $\%$. $78 \%$ of students say there is no communication between the two last ciclos. Por dimension to student participation in relation to the center $92 \%$ of students said they are not actively engaged and $59 \%$ say they do not promote a healthy environment.

Conclusion: The organization and management, and communication are negatively identified. We consider remaining design elements that illustrate this problem as central to the politics of academic management, to build joint resolution strategies.

\section{INTRODUCCIÓN}

Considerando el documento del Consejo Internacional de Enfermería sobre entornos saludables y su impacto en la calidad del cuidado, así como también las recomendaciones de la Organización Mundial de la Salud sobre promoción de la salud en el ámbito educativo, con la consecuente difusión de diferentes evidencias y resultados sobre la temática, se plantea la problemática tanto en el ámbito educativo como en el desempeño profesional. En el presente artículo se visualizan las primeras aproximaciones del entorno educativo de estudiantes de enfermería ${ }^{(1,2)}$

La relevancia del tema se centra en la preocupación por la influencia que el entorno significa para el compromiso de las enfermeras con la organización, hay evidencias al respecto que afirman que si el entorno es negativo contribuye a la ruptura del contrato psicológico entre el empleador y empleado y por consiguiente afecta la calidad del resultado. Por otro lado, diferentes autores coinciden en que un entorno saludable de trabajo es un contexto de la práctica profesional que favorece la salud y el bienestar de las enfermeras, la calidad del cuidado en los pacientes y los resultados de la organización. Un entorno laboral saludable es esencial, no sólo para lograr la salud de los trabajadores, sino también para hacer un aporte positivo a la productividad, la motivación laboral, el espíritu de trabajo, la satisfacción en el trabajo y la calidad ${ }^{(1,3,4)}$

La crisis mundial de los recursos humanos de enfermería caracterizada principalmente por escasez de enfermeras, muestra que los motivos de esa situación, son diversos, complejos, dentro de los cuales se observan los entornos de trabajo poco saludables. ${ }^{(5)}$

En el estudio del CIE sobre la crisis de los recursos humanos de enfermería; los entornos positivos para la práctica y los resultados de la organización fueron una de 
las cinco prioridades mundiales para la actuación. La Academia Estadounidense de Enfermería, identificó que en aquellos hospitales que las enfermeras señalaban al entorno de manera positiva, presentaban menores índices de agotamiento, mayor satisfacción en el trabajo y mejores resultados en los pacientes ${ }^{(6)}$. Estos hospitales fueron denominados en ese estudio como Hospitales Modelo, sus características pueden aplicarse a los entornos laborales, también a los lugares de formación de las enfermeras, pues no sólo posibilitarían su retención en los lugares de estudio, sino además, la posibilidad de aprender las características saludables de los entornos, para defender y construir en el futuros en sus lugares de trabajo ${ }^{(1)}$.

Otros estudios recomiendan para evaluar los entornos favorables y la práctica profesional algunas características, tales como: marcos de política general innovadores, centrados en la contratación y la retención; estrategias para una formación y perfeccionamiento profesional constantes; compensación adecuada; programas de reconocimiento; equipos y suministros suficientes y un entorno de trabajo seguro ${ }^{(1)}$.

En este sentido y considerando la etapa de formación de los enfermeros, es decir, el período de estudio de la carrera profesional, surge por parte del equipo de investigación la inquietud respecto a los entornos de estudio saludables, sabiendo que existen características que son comunes tanto a los lugares de trabajo como a los de estudio, tales como: calidad, elevados índices de retención, personal capacitado y experto en el área, horarios flexibles o alternativos que permitan una mayor correspondencia entre las necesidades de la persona y la oferta de la institución, entre otros.

Respecto a los espacios educativos, la evidencia respalda la importancia de ese ámbito, para generar y sostener acciones de promoción de salud que transforme a los mismos en entornos saludables. Una experiencia colombiana define que, "La Universidad Saludable debe formar personas creativas, seguras de sí mismas, críticas e innovadoras, debe crear valores de autoestima, autovaloración, asertividad, expresión de sentimientos, que les permitan enfrentar con seguridad las presiones del medio social, aumentar la capacidad productiva y el crecimiento espiritual, la capacidad de amar, jugar, gozar, relacionarse con otros, integrarse, crecer colectivamente y construir ciudadanía. Debe formar jóvenes independientes y respetuosos de los derechos humanos. Además, debe forjar un nuevo docente capaz de producir conocimientos e innovaciones en el campo educativo y pedagógico, crear condiciones agradables para el autoestudio y el autoaprendizaje, lograr que la apropiación de conocimientos ocurra en un ambiente democrático, de autoestima y solidaridad" ${ }^{2,7,8)}$.

Varios estudios, tanto teóricos como empíricos, señalan que el fracaso escolar y su consiguiente desgranamiento no tiene una única causa, ni tan siquiera un conjunto claramente definido que actúe de forma conocida, sino que las diferentes causas que se hipotetizan parecen variar en función del contexto del estudio, donde se identifican algunas categorías tales como: Factores organizacionales, Factores personales y familiares y Factores contextuales ${ }^{(9-12)}$.

Investigaciones señalan que los factores exclusivamente intelectuales y aptitudinales son pobres predictores del rendimiento académico a largo plazo y del éxito laboral fuera de los entornos educativos (Sternberg, Wagner, Williams \& Horvath, 1995). en el mejor de los casos los factores exclusivamente intelectuales explican alrededor de un 
$25 \%$ en la varianza del rendimiento académico, existiendo un alto porcentaje de variancia no explicada y por lo tanto atribuible a otros factores (Sternberg et al., 1995). En la actualidad, las investigaciones en este campo están volcadas a la búsqueda de factores no cognitivos que puedan explicar de forma más acabada el rendimiento académico ${ }^{(13)}$.

Recientes investigaciones han reconocido el aumento de los fracasos en el rendimiento académico de los alumnos en diferentes ámbitos académicos, así como también que la modificación de los entornos facilita el aprendizaje e incide en el fortalecimiento de los lazos grupales ${ }^{(2,7,14,15,)}$.

Considerando la argumentación previa, y los correlatos entre ambiente saludable y ámbito educativo; se eligió para una primera etapa de investigación, determinar los entornos de estudio y aprendizaje de los estudiantes de los cinco años de la carrera de Licenciatura en Enfermería de la Universidad Nacional de Córdoba. Las dimensiones del entorno estudiadas fueron: organización y gestión, dinámica vincular académica y participación estudiantil ${ }^{(19)}$.

Los factores institucionales son de gran importancia en estudios sobre factores asociados al rendimiento académico desde el punto de vista de la toma de decisiones, pues tienen que ver con variables, que en cierta medida se pueden establecer, controlar o modificar, por ejemplo, los horarios de los cursos, tamaños de grupos o criterios de ingreso en carrera.

Cabe señalar, que esta investigación es parte de una línea de investigación de Recursos Humanos en Salud, desarrollada en forma conjunta por las Cátedras de Enfermería en Salud Mental - Psiquiatría y de Enfermería de Alto Riesgo, de la Escuela de Enfermería de la Universidad Nacional de Córdoba, de la R Argentina.

\section{MATERIAL Y MÉTODO}

El tipo de estudio fue observacional, descriptivo de corte transversal.

La población estuvo compuesta por alumnos que cursaron la Licenciatura en Enfermería en el período 2011-2012, modalidad presencial y acreditaron al momento de la investigación su condición de alumno regular.

La muestra fue estratificada, aleatoria ( $\mathrm{n}: 290)$. La técnica utilizada fue una encuesta auto administrada y la fuente primaria.

\section{RESULTADOS}

Los resultados de esta etapa de la investigación son de carácter descriptivos, sin establecer aún relación entre variables. En cuanto a los datos demográficos el $46 \%$ de los alumnos poseen entre 18 y 25 años, el $44 \%$ entre 26 y 40 [Figura $N^{\circ} 1$ : Distribución etárea de los estudiantes de la Licenciatura de enfermería]. En relación al sexo, el $83 \%$ fueron mujeres, y el estado civil más representativo fue soltero, con el $68 \%$. 


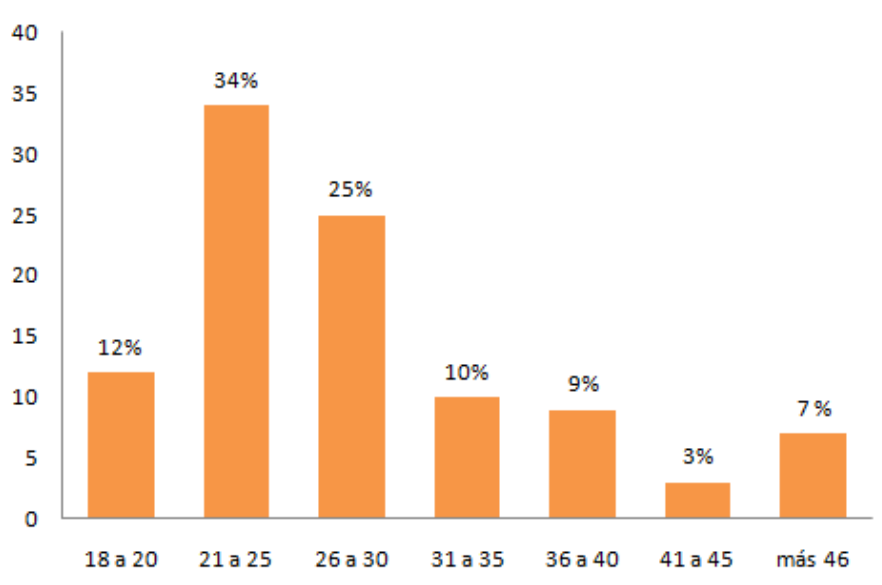

Figura $\mathrm{N}^{\circ}$ 1: Distribución etárea de los estudiantes de la licenciatura de enfermeria

En cuanto a la conformación de los grupos de convivencia, el $76 \%$ convive con la familia en primer lugar y en segundo lugar un $6 \%$ viven solos. El $69 \%$ del total de entrevistados poseen familiares a cargo; el $56 \%$ trabaja y el $44 \%$ recibe ayuda de los padres 0 son beneficiarios de becas estudiantiles que brindan cobertura a necesidades como alimentación, transporte y material bibliográfico.

Al analizar la dimensión organización y gestión del entorno, en la facilitación y uso de materiales para aprender las prácticas propias de la disciplina, se destaca que existe disponibilidad en más del $70 \%$ de los casos para desarrollar las mismas. El $62 \%$ de los alumnos señalan que no existe disponibilidad horaria flexible para el cursado. En cuanto a la facilitación de materiales en general, la distribución entre opiniones positivas y negativas es similar [Figura № 2: Materiales facilitados por la institución para el aprendizaje, según la opinión de los estudiantes].

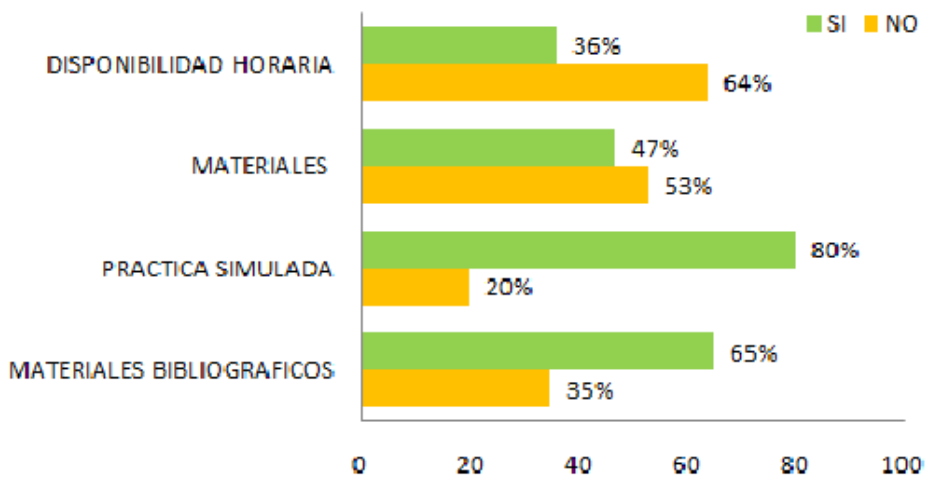

Figura $\mathrm{N}^{0} 2:$ Materiales facilitados por la institución para el aprendizaje, según la opinión de los estudiantes 
En la variable planta física, el 57\% de los alumnos respondió, que no es adecuada en lo referente a aulas, espacios de estudio y gabinetes. Identifican como ampliamente positiva la disponibilidad de la biblioteca, en un $79 \%$ de los casos y la iluminación en un 58\%. [Figura № 3: Disponibilidad y utilización de la planta física].

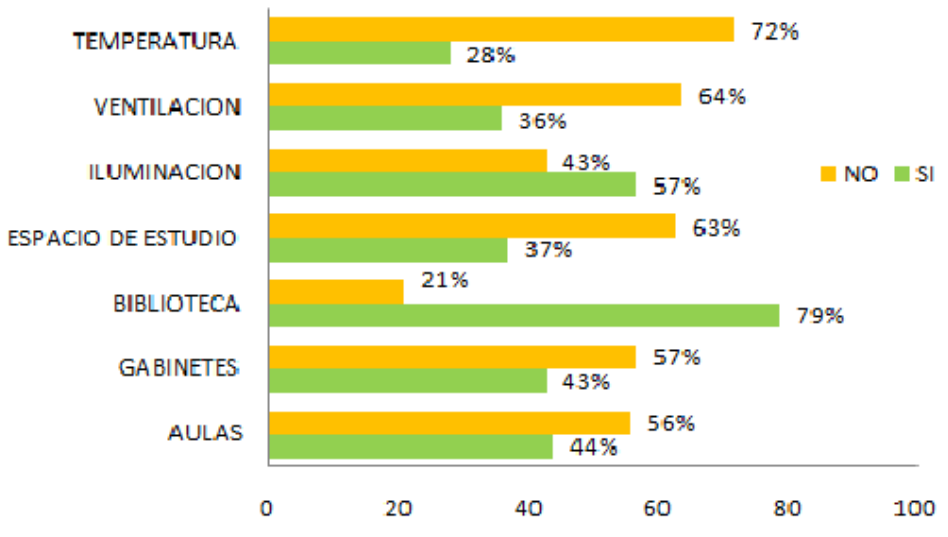

Figura $\mathrm{N}^{0}$ 3: Disponibilidad y utilización de la planta fisica

Los alumnos expresan que reciben apoyo en sus distintas variantes pedagógicas en valores superiores al $75 \%$, en este aspecto se hace referencia sobre todo a espacios de tutorías, asesoramiento y gabinete psicopedagógico en primer lugar. En relación a la disponibilidad del docente, esta es identificada en un porcentaje del $53 \%$. El acceso al material bibliográfico sugerido por los docentes y ofrecido en formato papel (libros y revistas), es valorado positivamente en un $75 \%$ de los estudiantes. [Figura $\mathrm{N}^{\circ} 4$ : Apoyo pedagógico a los estudiantes de la licenciatura durante el cursado de la carrera, según opinión vertida por los mismos].

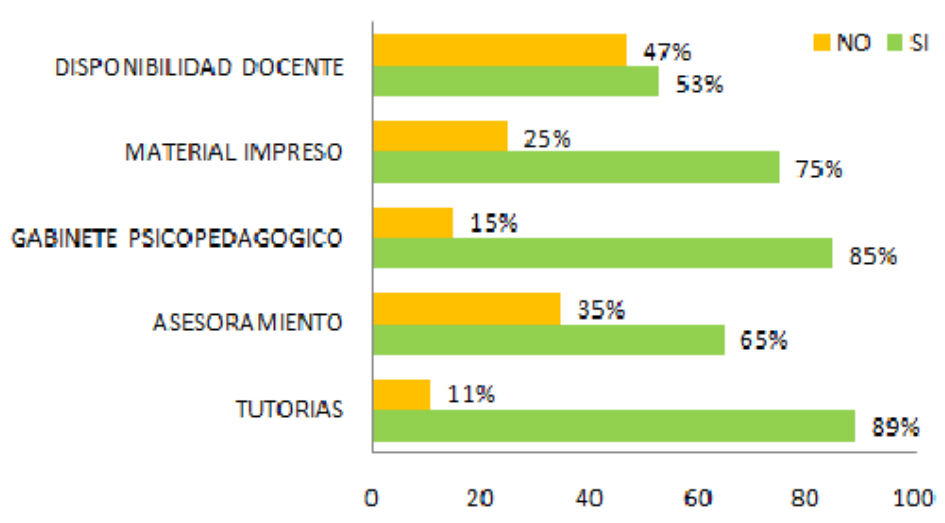

Figura $\mathrm{N}^{\circ}$ : Apoyo pedagógico a los estudiantes de la licenciatura durante el cursado de la carrera, según opinión vertida por los mismos

En cuanto a los procedimientos de formulación de quejas, el $75 \%$ respondió que sus reclamos no son escuchados por los directivos, con respecto a los reclamos referidos al personal no docente (áreas eminentemente administrativas y de gestión técnica) el 
$63 \%$ no son tenidos en cuenta, y en el caso del personal docente, el $44 \%$ no atiende los reclamos estudiantiles según los encuestados. El ámbito de mayor escucha es el centro de estudiantes donde un $75 \%$ señala que puede plantear quejas y reclamos [Figura 5: Atención a los reclamos de los estudiantes, según opinión de los estudiantes de la licenciatura de enfermería].

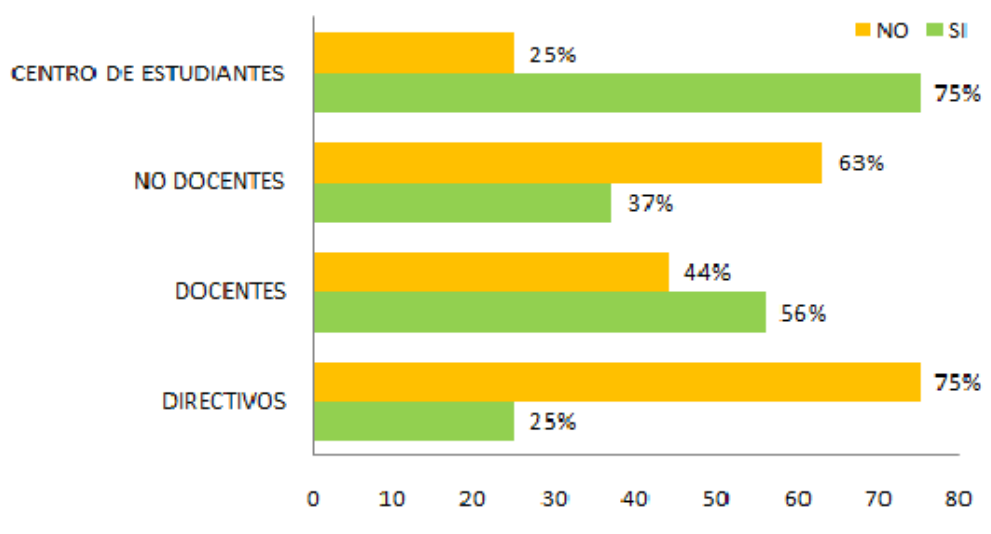

Figura 5: Atención a los reclamos de los estudiantes, según opinión de los estudiantes de la licenciatura de enfermeria

En la planificación de cátedras los alumnos respondieron en un $78 \%$, que la misma no existe, especificando que no hay una planificación acorde a las necesidades de los estudiantes, y en un $66 \%$ que no permite la planificación de la vida cotidiana, repercutiendo negativamente en el ámbito personal de cada estudiante [Figura 6: Aporte de la planificación de las cátedras a las necesidades de los estudiantes según opinión de los mismos]. El $52 \%$ de los encuestados respondió que la conducción en el período estudiado muestra debilidad para la gestión referida a las necesidades estudiantiles.

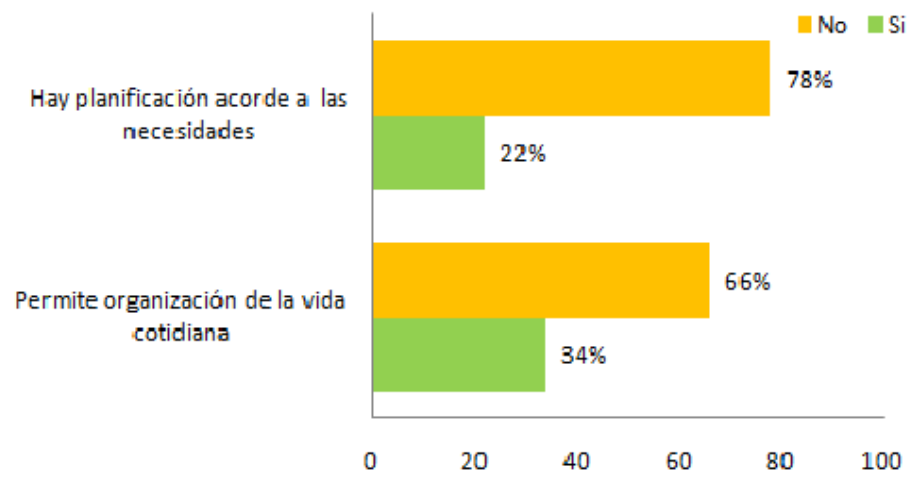

Figura 6: Aporte de la planificación de las cátedras a las necesidades de los estudiantes según opinión de los mismos 
Con respecto al aspecto vincular académico, los estudiantes expresan no sentirse escuchados en la institución en un $65 \%$, explicando que la comunicación entre pares es poca o medianamente colaborativa en un $59 \%$ de las veces. Por otro lado el $78 \%$ de los estudiantes afirman que no existe comunicación entre los dos ciclos, es decir, los alumnos que estudian en el primer ciclo para ser enfermeros técnicos profesionales y los que lo hacen en el segundo ciclo para la licenciatura de enfermería. [Figura 7 Aspecto vincular académico: Percepción de los estudiantes acerca de si se sienten escuchados en la institución].

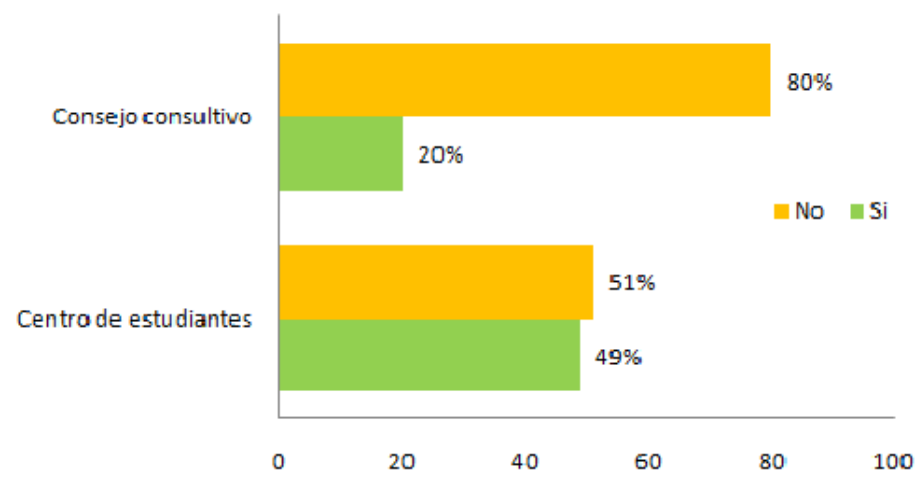

Figura 7 Aspecto vincular académico: Percepción de los estudiantes acerca de si se sienten escuchados en la institución

Por último, en la dimensión de participación estudiantil los estudiantes opinan mayoritariamente que no participan en forma activa en el centro de estudiantes, en un $92 \%$ de los casos y el $59 \%$ de los alumnos afirman que dentro de las acciones del centro de estudiantes no se promueve un entorno saludable.

\section{DISCUSIÓN}

Los resultados referidos a las características demográficas de esta investigación no se diferencian significativamente con los resultados generales en el ámbito de la Universidad Pública en dos estudios realizados en Universidades nacionales, siendo uno de ellos de nivel local. En los mismos se confirma la feminización del estudiantado y la prevalencia del grupo de 20 a 30 años ${ }^{(20,21)}$.

El porcentaje de alumnos con el beneficio de una beca registrado en este estudio se pueden relacionar con el documento del Ministerio de Salud de la Nación Argentina, en el cual se explican las políticas de incentivo a la carrera, tanto del ámbito nacional como provincial, para disminuir el déficit existente entre la cantidad de enfermeras graduadas y el aumento de demanda por parte del mercado de trabajo ${ }^{(22)}$.

Con relación a la dimensión organización y gestión, son identificados negativamente, tanto en sus aspectos más estructurales como son la planta física o la carga horaria de la carrera; como también en los aspectos dinámicos de la organización académica. En cuanto a los primeros se puede plantear que las dificultades señaladas respecto a la planta física se vinculan a la historia de la escuela de enfermería, pues el espacio físico propio de la institución es el mismo desde su fecha de fundación 1954 y no se 
ha expandido, a pesar de haber aumentado la matrícula según se consigna en estudios de seguimiento realizados por el Programa de Estadísticas Universitarias ${ }^{(21)}$

Otro hallazgo de esta investigación con característica negativa en los entornos educativos es la utilización de aulas comunes a estudiantes de otras carreras y en diferentes lugares del campus universitario. Esto es considerado en un estudio realizado en Colombia como un factor potencial de riesgo pues implica el desplazamiento de los estudiantes en diferentes espacios físicos que no siempre coinciden con la disponibilidad de los servicios de apoyo en ese mismo ámbito, (biblioteca o laboratorio de prácticas simuladas). Estas características que son propias de la institución estudiada significan a su vez disminución del tiempo útil, por la pérdida del mismo en los desplazamientos ${ }^{(23) .}$

Diferentes autores refieren que los aspectos estructurales de una organización pueden flexibilizarse para posibilitar procesos dinámicos más acordes con las demandas de los estudiantes, así se potenciaría el impacto pedagógico en los estudiantes. Los autores mencionados aportan además a la discusión el rol del personal, en este caso docente y no docente, en su relación burocrática con el estado y su participación en la continuidad e innovación de las políticas. Estos aspectos en el presente estudio se pueden visualizar en la dimensión referida a la gestión y específicamente a los materiales facilitados por la institución para el aprendizaje y los cronogramas de horarios donde se desarrolla a la actividad académica, los cuales son evaluados como poco flexibles por los estudiantes ${ }^{(24-29)}$

Según los resultados, los estudiantes observan la necesidad de flexibilizar la dinámica interna de la escuela de enfermería y generar espacios educativos saludables, ya que no es una política institucional que atraviese los distintos claustros. Las experiencias latinoamericanas de Costa Rica y Colombia pueden ser una base de transformación en la institución estudiada.

Estos aspectos se relacionan también con la dimensión vincular académica, no sólo porque se condicionan mutuamente sino porque generan visiones similares en cuanto al factor de la comunicación, que muestra dificultades entre los estudiantes, en la relación con los docentes y no docentes.

En cuanto a la dimensión de participación estudiantil, los datos refieren debilidad en las distintas esferas universitarias. Los mismos, se vinculan con hallazgos de otros estudios que expresan que los procesos sociales propios del postmodernismo tienen lazos sociales débiles y la participación ciudadana es principalmente delegativa. El fortalecimiento de los mecanismos de participación no implica en absoluto el debilitamiento de las instituciones de representación, por el contrario, requiere su fortalecimiento simultáneo ${ }^{(30)}$. (Discusiones en este sentido pueden profundizarse en autores como: Prats, Joan (1999) Reinventar la burocracia y construir la nueva gerencia pública, España: Instituto Internacional de Gobernabilidad, colección documentos www.iigov.org.-. Ramió Matas, Carles "Los problemas de la implantación de la nueva gestión pública en las administraciones pública latinas: modelo de estado y cultura institucional" Reforma y democracia 21, octubre de 2001, clad, Caracas Venezuela.-. Sennett Richard "La corrosión del carácter, las consecuencias personales del trabajo en el nuevo capitalismo" editorial Anagrama, España 2000). 


\section{CONCLUSIONES}

Respecto a las características generales del grupo estudiantil, se concluye que su conformación no difiere de la general universitaria y a la histórica de la carrera.

En cuanto al contexto educativo, especialmente a la dimensión organización y gestión, se identifica la necesidad de mejorar las mismas para acercar la institución universitaria a parámetros de ambientes saludables, considerando que hay aspectos altamente positivos como el apoyo docente en sus distintas metodologías pedagógicas.

En la dimensión vincular académica y en la de participación estudiantil, ambas, son débiles en las distintas esferas universitarias, siendo recomendable diseñar elementos que visibilicen esta problemática como central en la política de gestión académica, para construir estrategias conjuntas de resolución.

\section{REFERENCIAS BIBLIOGRÁFICAS}

(1) Consejo Internacional de Enfermeras (CIE). Entornos de práctica favorables: lugares de trabajo de calidad = atención de calidad al paciente. Ginebra - Suiza 2007. [Acceso marzo 2014] Disponible en: http://www.icn.ch/images/stories/documents/ publications/ ind/indkit2007sp.pdf

(2) Ministerio de Protección social Ministerio de Ambiente, Vivienda y Desarrollo territorial; ops/Colombia. Entornos saludables: Guía del Docente. Bogotá. 2006. [Acceso marzo 2014] Disponible en: http://www.bvsde.paho.org/bvsdeescuelas/fulltext/entornosdocente/presenta.pdf

(3) Casas SB, Klijn TP. Promoción de la salud y su entorno laboral saludable. Rev Latinoamericana Enfermagem 2006 janeiro- fevereiro; 14(1) : 136- 41. [Acceso marzo 2014] Disponible en: http://www.eerp.usp.br/rlae

(4) Organización Panamericana de la Salud. Organización Mundial de la Salud. División de Salud y Ambiente. Programa Regional de Salud de los Trabajadores. Documento de Trabajo. Anexo № 6: Estrategia de Promoción de la salud en los lugares de trabajo de América Latina y el Caribe. San José, Costa Rica, Marzo 15 a 17 de 2000. [Acceso marzo 2014] Disponible en: http://www.who.int/occupational health/regions/en/oehpromo cionsalud.pdf

(5) Organización Panamericana de la Salud. Metas Regionales en Materia de Recursos Humanos para la Salud 2007-2015. 27 Conferencia sanitaria Panamericana, 59 Sesión del Comité Regional de la OMS para las Américas. 2007. WDC.[Acceso en Marzo 2014] Disponible en: http://www.paho.org/sapanich/gov/.pdf.

(6) Aiken L., Clarke S., Sloane D., Soalski J., Silber J. Enfermera del hospital Dotación y mortalidad de los pacientes, la enfermera de la quemadura, y la insatisfacción laboral. JAMA 2002; 288 (16) :1987-1993. doi: 10.1001/jama.288.16.1987. [Acceso marzo 2014] Disponible en: http://jama.jamanetwork.com/article.aspx?articleid=195438

(7) OPS/OMS, Boletín No. 5 de la Red Colombiana de Escuelas Saludables por la Paz. [Acceso marzo 2014] Disponible en: http://www.minproteccionsocial.gov.co/ entornoambiental/library/documents/DocNewsNo15493DocumentNo3280.pdf

(8) Giraldo Osorio A., Toro Rosero M.Y., Macías Ladino A.M., Valencia Garcés C. A., Palacio Rodríguez S. La promoción de la salud como estrategia para el fomento de estilos de vida saludables. Volumen 15, No.1, enero - junio 2010, págs. 128 - 143. [Acceso marzo 2014] Disponible en http://promocionsalud.ucaldas.edu.co/ downloads/ Revistas15(1)_9.pdf 
(13) Lozano Díaz A. Factores personales, familiares y académicos que afectan al fracaso escolar en la educación secundaria. Almería, España. 2003. Revistra welectrónica de investigación psicoieducativa y psicopedagógica, 1(1),43-66. [Acceso marzo 2014] Disponible en www.investigacionpsicopedagogica.org/revista/articulos/1espannol/Art_1_4.pdf

(14) Robledo Ramón P. y García Sánchez J.N. El entorno familiar y su influencia en el rendimiento académico de los alumnos con dificultades de aprendizaje: revisión de estudios empíricos. Aula abierta 2009, vol 37, n 1, pp117-128, ICE. Universidad de Oviedo.Espana. [Acceso en marzo 2014] Disponible en: http://www.minproteccionsocial.gov.co/entornoambiental/library/documents/DocNews No15493DocumentNo3280.pdf

(15) Garbanzo Vargas G.M. Factores asociados al rendimiento académico en estudiantes universitarios, una reflexión desde la calidad de la educación superior pública. Revista Educación 31(1), 43-63. 2007. [Acceso en marzo 2014] Disponible en: http://latindex.ucr.ac.cr/index.php/educacion/article/viewFile/1252/1315

(16) Marchesi A., Martinez R., Matin E. Estudio Iongitudinal sobre la influencia del nivel socio cultural en el aprendizaje de los alumnos en la educación secundaria obligatoria. Infancia y aprendizaje. 2004. 27(3), 307-323.

(17) Beguet, Bernardo. Factores que intervienen en el rendimiento académico de los estudiantes de psicología y psicopedagogía. Universidad de El Salvador.

(18) Herrera Batista, M.A. Las Nuevas Tecnologías en el Aprendizaje Constructivo. Universidad Autónoma Metropolitana, México. La Revista Iberoamericana de Educación. Numero 34/4. [Acceso en marzo 2014] Disponible en: http://www.rieoei.org/deloslectores/821Herrera.PDF

(19) Mariño S. I. y Godoy M. V. Desarrollo de entornos virtuales educativos. Contribuciones desde el Área de Ingeniería Web. Revista electrónica, Quaderns Digitals/Quaderns número 53. [Acceso en marzo 2014] Disponible en: http://www.quadernsdigitals.net/index.php?accionMenu=hemeroteca.VisualizaArticulol U.visualiza\&articulo id $=10492$

(20) Di Gresia L., Porto A. y Ripani L. Rendimiento de los Estudiantes de las Universidades Públicas Argentinas Documento de Trabajo Nro. 45. Noviembre 2002. Departamento de Estadísticas. Facultad de Ciencias Económicas. Universidad Nacional de la Plata. [Acceso en marzo] Disponible en: http://www.depeco.econo.unlp.edu.ar/doctrab/doc45.pdf

(21) Goldenhersch H., Llinás G., Balzarini M., Maccagno A., Somazzi C., Esbry N. Espacio lberoamericano del Conocimiento. Dos siglos de graduaciones en la UNC registros históricos y experiencias del presente. Secretaría de Asuntos Académicos Programa de Estadísticas Universitarias. Universidad Nacional de Córdoba, 2010. [Acceso en marzo] Disponible en: http://www.chubut.edu.ar/descargas/secundaria/congreso/EIC/R0327 Maccagno.pdf (22) Ministerio de Salud. Presidencia de la Nación. Proyecto De Ley de Emergencia de Enfermería Consejo Federal de Salud. 12 de Setiembre de 2008. [Acceso en Marzo 2014] Disponible en: http://www.msal.gov.ar/images/stories/cofesa/2008/acta03-08/anexo-12-03-08.pdf

(23) Machado Alba, J. E. La Universidad Saludable. Revista de Ciencias humanas No 16, Julio de 2001. [Acceso 4 de marzo de 2014] Disponible en: http://www.utp.edu.co/ chumanas/revistas/revistas/rev26/machado.htm

(24) Mintzberg, H. Diseño de organizaciones eficientes. Cátedra Administración gerencial. UTN-FRSFD. 2001. [Acceso marzo de 2014] Disponible en: file:///C:/Users/saludpublica/Downloads/Apunte Dise\%C3\%B10 Organiz Final.pdf

(25) Hintze, J. Administración de Estructuras Organizativas. Revista trasformación, estado y democracia 37. 67-106 [Acceso en febrero 2011] Disponible en: 
http://www.onsc.gub.uy/onsc1/images/stories/Publicaciones/RevistaONSC/r37/378.pdf

(26) Hammer, M. y Stanton, S. La Revolución de la reingeniería Reingeniería. Ediciones Díaz de Santos, 1997 -384 páginas.

(27) Universidad de Alicante. Red española de universidades saludables (REUS). La universidad de Alicante: Una Universidad saludable.2008. [Acceso en Marzo 2014] Disponible en: http://web.ua.es/es/sep/documentos/pdf/ua/red-espanola-deuniversidades-saludables.pdf

(28) Hintze, J. Gestión por procesos y por resultado en el aparato estatal; una cuestión abierta. Ponencia presentada en el Segundo Congreso Argentino de Administración Publica. 2003. República Argentina. [acceso marzo de 2014] Disponible en: http://issuu.com/asap1/docs/gestionporprocesosyresultados?e=3399398/2439275\#se $\underline{\operatorname{arch}}$

(29) Carvalho R.S, Viana L, La formación del enfermero en las escuelas de enseñanza media desde un concepto de interdisciplinariedad. Vol 8, Núm 1 (2009) [Acceso $15 \quad$ Febrero 2014] Disponible en http://revistas.um.es/eglobal/article/view/49812/47692

(30) Ase, I. El futuro de la modernización administrativa del estado: de la ilusión tecnocrática a la reconstrucción de lo público. Segundo Congreso Argentino de Administración Pública. Sociedad, Estado y Administración. 2002. [Acceso en marzo de 2014] Disponible en: http://aaeap.org.ar/wp-content/uploads/2013/03/Ase Ivan.pdf

Recibido: 9 de mayo 2014; Aceptado: 11 de junio 2014

ISSN 1695-6141

(C) COPYRIGHT Servicio de Publicaciones - Universidad de Murcia 